DESY 98-186

\title{
Gravitino constraints on models of neutrino masses and leptogenesis
}

\author{
David Delepine ${ }^{1}$, Utpal Sarkar ${ }^{2}$ \\ ${ }^{1}$ DESY, Notkestrasse 85, D-22607 Hamburg, Germany \\ 2 Physical Research Laboratory, Ahmedabad 380 009, India
}

\begin{abstract}
In the supersymmetric extensions of the standard model, neutrino masses and leptogenesis requires existence of new particles. We point out that if these particles with lepton number violating interactions have standard model gauge interactions, then they may not be created after reheating because of the gravitino problem. This will rule out all existing models of neutrino masses and leptogenesis, except the one with right-handed singlet neutrinos.
\end{abstract}


Recent announcement of a positive evidence for a neutrino mass from an observation of the atmospheric neutrino anomaly [1] and the supporting evidence from the solar neutrino puzzle [2] implies an extension of the standard model. In all the models of neutrino masses considered so far, require existence of new heavy particles $H$ with a mass $M$ and lepton number violating interactions. At low energy this results in an effective dimension-5 operator $\mathcal{O}=h^{2} \frac{1}{M} \ell_{L} \ell_{L} \phi \phi$, where $\phi$ is the usual higgs doublet which gives masses to the quarks and charged leptons. When $\phi$ acquires a vacuum expectation values (vev) to break the electroweak symmetry, the left-handed neutrinos get small Majorana masses, $m_{\nu}=\frac{(h\langle\phi\rangle)^{2}}{M}$. $H$ could be a $S U(2)_{L}$ singlet or a triplet and it could be a fermion or a scalar, which gives four possible categories of models for neutrino masses [3].

In all these models the decays of the new particles $H$ violate lepton number and can, in general, generate a lepton asymmetry of the universe, which then gets converted to a baryon asymmetry of the universe before the electroweak phase transition 4 . The out-of-equilibrium distribution of $H$ requires $M$ to be very heavy, particularly if $H$ has got standard model gauge interactions. In this article we point out that if we constrain the reheating temperature after inflation by requiring that the universe is not overpopulated by unacceptably large number of gravitinos, then that singles out models with right handed neutrinos without any standard model gauge interactions to be the only consistent class of model for neutrino masses and leptogenesis. In the following we first discuss the different models and then discuss the gravitino constraint.

Right handed neutrinos : The fermion content of the standard model is extended to include one right handed neutrinos $\left(N_{\alpha}, \alpha=1,2,3\right)$ per generation, which are singlets under the standard model gauge group. The Majorana masses and the Yukawa couplings of the right-handed neutrino 
with other leptons are given by,

$$
\mathcal{L}=M_{\alpha} \overline{N_{\alpha}^{c}} N_{\alpha}+h_{i \alpha} \overline{\ell_{i L}} N_{\alpha} \phi+h . c . .
$$

Without loss of generality we assume that the right handed neutrino Majorana mass matrix is real and diagonal. The lepton number violating scale is then given by the right-handed neutrino masses, $M_{\alpha}$. When the higgs doublet $\phi$ acquires a vev, $\langle\phi\rangle=v$, there will be an induced Dirac mass matrix for the neutrinos, $\left(m_{D}\right)_{i \alpha}=h_{i \alpha} v$. By the usual see-saw mechanism[5], the left-handed neutrinos get a small Majorana mass $M_{\nu}=-m_{D}^{T} \frac{1}{M_{R}} m_{D}$.

The decay of the right handed neutrino $N_{\alpha}$ now breaks lepton number. $C P$ violation comes from an interference of the vertex and self-energy oneloop diagrams with the tree level diagrams [6, 7] and the amount of asymmetry is given by,

$$
\epsilon_{\alpha} \simeq \frac{3}{16 \pi v^{2}} \sum_{\beta \neq \alpha} \frac{1}{\left(m_{D}^{\dagger} m_{D}\right)_{\alpha \alpha}} \operatorname{Im}\left[\left(m_{D}^{\dagger} m_{D}\right)_{\alpha \beta}^{2}\right] \frac{M_{\alpha}}{M_{\beta}}
$$

when $M_{\alpha}<M_{\beta}$ and $N_{\alpha}$ is the lightest right handed neutrino. The decay of $N_{\alpha}$ can generate this amount of lepton asymmetry of the universe, if it satisfies the out-of-equilibrium condition, $\Gamma_{N_{\alpha}}=\frac{h_{i \alpha}^{2}}{8 \pi} M_{\alpha}<H=1.7 \sqrt{g_{*}} \frac{T^{2}}{M_{P l}} \quad$ at $T=$ $M_{\alpha}$, where, $H$ is the Hubble constant, $g_{*}$ is the effective number of helicity states, $M_{P l}$ is the Planck scale.

Triplet higgs : It is possible to extend the standard model to include $S U(2)_{L}$ triplet higgs scalar fields $\left(\xi_{a} \equiv(1,3,-1), a=1,2\right.$ two of them are required for $C P$ violation), whose relevant interactions are,

$$
\mathcal{L}=M_{a} \xi_{a}^{\dagger} \xi_{a}+f_{i j} \xi_{a}^{\dagger} \ell_{i} \ell_{j}+\mu \xi_{a} \phi \phi+h . c .
$$

We choose $M_{a}$ to be real and diagonal. The parameter $\mu$ has a dimension of mass and is of the order of $M_{a}$, which is the only scale in this model other than the electroweak symmetry breaking scale. Lepton number is explicitly broken at this scale, but the triplet higgs acquires a very tiny vev, which 
gives a Majorana mass to the neutrinos [8, 9], $M_{\nu}=f_{i j}<\xi_{a}>=-f_{i j} \frac{\mu<\phi\rangle^{2}}{M_{a}^{2}}$ . Lepton number violation comes from the decays of the triplet higgs, $\xi_{a}$. In this case there are no vertex corrections to these decay processes. The one loop self-energy diagrams interfere with the tree level decays to give $C P$ violation. If $M_{2}>M_{1}$ and $\xi_{1}$ decays away from thermal equilibrium, i.e., $\Gamma_{1}=\frac{1}{8 \pi M_{1}}\left(\mu_{1} \mu_{1}^{*}+M_{1} M_{1} \sum_{k, l} f_{1 k l}^{*} f_{1 k l}\right)<H=1.7 \sqrt{g_{*}} \frac{T^{2}}{M_{P l}} \quad$ at $T=M_{1}$, then a lepton asymmetry will be generated [8],

$$
\frac{n_{L}}{s} \simeq \frac{\operatorname{Im}\left[\mu_{1} \mu_{2}^{*} \sum_{k, l} f_{1 k l} f_{2 k l}^{*}\right]}{24 \pi^{2} g_{*}\left(M_{1}^{2}-M_{2}^{2}\right)}\left[\frac{M_{1}}{\Gamma_{1}}\right] .
$$

Triplet Majorana fermions : One can also extend the standard model to include a $S U(2)_{L}$ triplet fermions, whose large Majorana masses breaks lepton number. For all practical purposes they behave similar to those like the right handed neutrinos and give neutrino masses through see-saw mechanism [10]. Their decay can generate a lepton and hence baryon asymmetry of the universe in the same way as that of a singlet right handed neutrino.

Radiative models : It is possible to write down the effective dimension5 operator with $S U(2)_{L}$ singlet field $\left(\chi_{a} \equiv(1,1,-1), a=1,2\right.$, two of these fields are required to get $C P$ violation) if there are at least two higgs doublets $\left(\phi_{a}, a=1,2\right)$. The neutrino masses originate from radiative diagrams and hence are naturally small [11]. The charged singlet scalar $(\chi)$ have couplings to two higgses and also to two leptons of two different generations, thereby breaking the lepton number explicitly,

$$
\mathcal{L}=M_{a}^{\chi} \chi_{a}^{\dagger} \chi_{a}+f_{a i j}^{\chi} \chi_{a}^{\dagger} \ell_{i} \ell_{j}+\mu_{\chi} \chi_{a} \phi_{1} \phi_{2}+\text { h.c. }
$$

Unlike the triplet scalar case, $\chi$ cannot acquire a vev and hence neutrino masses can come only radiatively. Although this model has been studied in details for neutrino masses and has been found to have several interesting features in the context of the present experiments [12, the question of 
leptogenesis is only recently being investigated [13]. Without going into the details we make here a few general comments about this model.

Since the decays of this charged scalar violates lepton number in a similar way to that of the triplet scalars, a lepton asymmetry can be generated in the same way. The amount of asymmetry generated is also similar, except that the parameters involved are now changed. The main point we like to stress here is that the couplings of $\chi_{a}$ violate lepton number at a very high scale. Since these singlets are charged, they also suffer from the same problem as the triplets that it interacts very fast with the standard model gauge bosons. So all the constraints coming due to the gauge interactions of the particles whose decay generates an asymmetry will also be applicable to this case.

Among all the above four classes of models for neutrino masses, only models with a singlet right-handed neutrino does not have any standard model gauge interaction. In all the three other classes of models, the new particles whose interactions break lepton number, transform non-trivially under the standard model. We shall next study the consequences of the standard model gauge interaction on the generation of a lepton asymmetry of the universe when these particles decay.

For simplicity, we shall consider a couple of generic heavy scalar $H_{a}, a=$ 1,2 , which couples to the standard model gauge bosons through gauge interactions. In a supersymmetric model, the corresponding superpartner will have similar gauge interactions with the gauginos and hence will suffer from the same problem. The generic scalar $H_{a}$ could be $\xi_{a}$ as in the triplethiggs model, in which case it will interact with the $S U(2)_{L}$ and $U(1)_{Y}$ gauge bosons, or it could be $\chi_{a}$ as in the Zee-type radiative models, in which case it will interact only with the $U(1)_{Y}$ gauge bosons. For the generation of a lepton asymmetry of the universe we assume that the relevant part of the lagrangian is similar to that of eqn(6). We choose $M_{a}^{h}$ to be real and diagonal and $\mu^{h} \lesssim M_{a}^{h}$. Decay of $H_{a}$ into two leptons and two higgs together violate lepton number. The tree level and the one loop self energy diagrams interfere 
to generate a $C P$ asymmetry $\eta$. We shall also assume that $M_{1}^{h}<M_{2}^{h}$, so that first $M_{2}^{h}$ decays and then the decay of $M_{1}^{h}$ generates the lepton asymmetry of the universe.

The evolution of lepton number $\left(n_{L}=n_{\ell}-n_{\ell^{c}}\right)$ is given by the Boltzmann equation [14,

$$
\mathcal{D} n_{L}=\eta \Gamma_{H}\left[n_{H}-n_{H}^{e q}\right]-\left(\frac{n_{L}}{n_{\gamma}}\right) n_{H}^{e q} \Gamma_{H}-2 n_{\gamma} n_{L}\left\langle\sigma_{L}|v|\right\rangle,
$$

where, the operator $\mathcal{D} \equiv\left[\frac{\mathrm{d}}{\mathrm{d} t}+3 H\right] ; n_{H}^{e q}$ is the equilibrium distribution of $H_{1}$ given by $n_{H}^{e q}=\frac{T M_{1}^{h 2}}{2 \pi^{2}} K_{2}\left(\frac{M_{1}^{h}}{T}\right) ; \Gamma_{H}$ is the thermally-averaged decay rate of $H_{a} ; n_{\gamma}$ is the photon density and $\left\langle\sigma_{L}|v|\right\rangle$ is the thermally-averaged lepton number violating scattering cross section.

The number density $\left(n_{H}\right)$ of $H_{1}$ satisfies the Boltzmann equation,

$$
\mathcal{D} n_{H}=-\Gamma_{H}\left(n_{H}-n_{H}^{e q}\right)+\left(n_{H}{ }^{2}-n_{H}^{e q 2}\right)\left\langle\sigma_{H}|v|\right\rangle .
$$

The second term on the right is the lepton number conserving thermallyaveraged $H_{1}^{\dagger}+H_{1} \rightarrow W_{L}+W_{L}$ scattering cross section of the heavy particles $H_{1}$. It is instrumental in initially equilibrating the number density of $H_{1}$ (which will also erase any lepton asymmetry created during the decay of $H_{2}$ ) but it also prevents departure from equilibrium for $H_{1}$, which depletes the generated lepton asymmetry if this interaction becomes comparable to the expansion rate of the universe.

To solve these equations, we use the dimensionless variable $x=M_{1}^{h} / T$, and normalize the particle density by the entropy density, $Y_{i}=n_{i} / s$, so $t=$ $x^{2} / 2 H(x=1)$. We also define the parameters $K \equiv \gamma \Gamma_{H(x=1)} / H_{(x=1)} ; K_{H} \equiv$ $\gamma_{H}\left\langle\sigma_{H}|v|\right\rangle_{(x=1)} / H_{(x=1)} ; \gamma=\Gamma_{H} / \Gamma_{H(x=1)} ; \gamma_{H}=\left\langle\sigma_{H}|v|\right\rangle /\left\langle\sigma_{H}|v|\right\rangle_{(x=1)}$ and $\gamma_{s}=$ $n_{\gamma}\left\langle\sigma_{L}|v|\right\rangle / H_{(x=1)}$. If the out-of-equilibrium condition is satisfied $(K \ll 1)$, the final lepton asymmetry is given by $n_{L} \sim \eta / g_{*}$, if there is no suppression due to the scattering. Since we are interested in the maximum amount of possible lepton asymmetry in this scenario, we shall always consider the 
couplings of $H_{a}$, which satisfies this out-of-equilibrium condition, $K \ll 1$ at $T \sim M_{1}^{h}$. However, we do not have this freedom for the scattering processes, since the standard model gauge coupling constants are involved. We solve these two nonlinear equations numerically. To get an unsuppressed lepton asymmetry we work with $K \ll 1$. If we ignore the lepton number conserving gauge interactions of $H_{1}$, the lepton asymmetry is now given by $n_{L} \sim \eta / g_{*}$. At temperatures of interest, i.e., after the inflation, the restrictions imposed on the couplings of $H_{a}$ by $K \ll 1$ will not allow $\eta$ to be larger than $\sim O\left(10^{-5}\right)$.

For our analysis we shall thus assume, $K \ll 1$ at $T \sim M_{1}^{h}$ and $\eta<10^{-5}$. Taking the $S U(2)_{L}$ gauge coupling constant to be given by the GUT coupling constant at the GUT scale, we include the effect of the lepton number conserving gauge interactions of $H_{1}$ and present it in figure 1 .

For very high $M_{1}^{h}$ masses around the GUT scale, when $K_{H} \ll K$ the lepton asymmetry is given by $n_{L} \sim \eta / g_{*}$. As we lower $M_{1}^{h}$ masses, the scattering processes become comparable to the expansion rate of the universe and start depleting the amount of lepton asymmetry. For $K_{H} \sim K$, we already get a suppression by two orders of magnitude. So, for the allowed value of $\eta \leq 10^{-5}$ the lowest possible $H_{1}^{h}$ mass for the generation of enough lepton asymmetry of the universe becomes

$$
M_{1}^{h}>O\left(10^{12}\right) \mathrm{GeV}
$$

The above conclusion will also be true for the triplet Majorana fermion models, where the lepton number violation in induced by the Majorana mass of the Majorana triplet fermion $T$. The gauge interactions will then induce a lepton number conserving scattering process $T^{\dagger}+T \rightarrow W_{L} \rightarrow T^{\dagger}+T$, which will deplete the lepton asymmetry.

We have to keep in mind that leptogenesis can occur only after the end of inflation. In supersymmetric theories, the thermal production of massive gravitinos restricts the beginning of the radiation-dominated era following inflation except when the gravitino is very light[15]. After the inflation a 
large number of gravitinos are produced, which interact very weakly. The late decays of unstable gravitinos can then modify the abundances of light elements causing inconsistency with observation. In the other hand, stable gravitinos may overclose the universe. This imposes a upper bound on the reheating temperature $T_{R H}$ [16, 17, 18, 20].

In the case of stable gravitinos, a limit on the $T_{R H}$ can be derived from the closure limit of the universe [19],

$$
T_{R H} \leq 10^{10} \mathrm{GeV} \times\left(\frac{m_{3 / 2}}{100 \mathrm{GeV}}\right) \times\left(\frac{1 \mathrm{TeV}}{m_{\tilde{g}}(\mu)}\right)^{2}
$$

with $m_{3 / 2}$ is the mass of the gravitino and $m_{\tilde{g}}(\mu)$ is the running mass of the gluino. An upper bound on the gravitino mass also arises when we are taking into account the effect of the decays of the next-to-lightest superparticle (NSP) on primordial nucleosynthesis. For instance, it was shown in Ref. 19 than for a $m_{3 / 2}=1 \ldots 10^{3} \mathrm{GeV}$ and $80 \mathrm{GeV}<m_{N S P}<300 \mathrm{GeV}$, a $T_{R H}$ as large as $10^{8-11} \mathrm{GeV}$ could be reached.

In the case of unstable gravitinos, the upper bound on the $T_{R H}$ depends on the $m_{3 / 2}$. Essentially, one gets the followings constraints from primordial nucleosynthesis 21

$$
\begin{array}{rr}
T_{R H} \leq 10^{9} \mathrm{GeV} & m_{3 / 2}<1 \mathrm{TeV} \\
T_{R H} \leq 10^{12} \mathrm{GeV} & 1 \mathrm{TeV}<m_{3 / 2}<5 \mathrm{TeV}
\end{array}
$$

It has recently been shown that after the reheating temperature $\left(T_{R H}\right)$, inflaton decay can produce particles as heavy as $\sim 10^{3} T_{R H}$ with sufficiently large abundances 22]. In that case, they get a suppression factor coming from the annihilation cross section of the heavy particles, which is the scattering term in the Boltzmann equation (7). Thus the suppression factor is similar to the suppression due to the departure of the scattering process from equilibrium. In other words, particles with mass 10 times $T_{R H}$ will have an abundance about $S \sim O\left(10^{-3}\right)$ times less than the equilibrium abundance, 
which can generate a lepton asymmetry which is less by a factor of $S$. So, even for the stable gravitino, when the bound on the reheating temperature is given by eqn(9), it will not be possible to generate enough lepton asymmetry in these scenarios, where the lepton number violating particle have got standard model gauge interactions.

In the left-right symmetric models [23], both the right-handed neutrinos and the triplet higgs are present. However, in most of the realistic models the triplet higgs are much heavier than the right handed neutrinos and a lepton asymmetry is generated when the lightest right handed neutrino decay. In SO(10) GUT the left-right symmetric models are naturally embedded, in which it has been shown that the mass of the lightest right handed neutrino comes out to be just consistent with the gravitino bound and on the other hand the amount of generated lepton asymmetry is also as required [24]. This may indicate that the recent positive evidence of the neutrino masses may actually directing us towards a supersymmetric $S O(10)$ GUT.

In summary, we point out that all the supersymmetric models of neutrino masses, except for the one with singlet right handed neutrinos and left-right symmetric models, may not be able to generate enough lepton asymmetry of the universe consistently with the gravitino bound on the reheating temperature in inflationary universe. So see-saw mechanism of the neutrino masses with right handed singlet neutrinos and left-right symmetric models, such as supersymmetric $S O(10)$ or superstring inspired $E(6)$ GUTs, then becomes the most preferred solution for neutrino masses and leptogenesis.

Acknowledgements We thank Prof. W. Buchmüller for useful comments and discussions.

\section{References}


[1] Super-Kamiokande Collaboration : Y. Fukuda et al, Phys. Rev. Lett. 81, 1562 (1998); hep-ex/9805006; Phys. Lett. B433, 9 (1998); T. Kajita, hep-ex/9810001.

[2] Super-Kamiokande Collaboration: Y. Fukuda et al, Phys. Rev. Lett. 81, 1158 (1998); Talk by Y. Suzuki at Neutrino'98, Takayama, Japan (1998).

[3] E. Ma, hep-ph/9807386, Phys. Rev. Lett. 81, 1171 (1998) (hepph/9807386).

[4] S. Yu. Khlebnikov and M.E. Shaposhnikov, Nucl. Phys. B 308, 885 (1988); J.A. Harvey and M.S. Turner, Phys. Rev. D 42, 3344 (1990).

[5] M. Gell-Mann, P. Ramond and R. Slansky, in Supergravity, eds. P. van Nieuwenhuizen and D. Freedman, (North-Holland, 1979) p.315; T. Yanagida, in Proc. Workshop on Unified Theories and Baryon Number in the Universe, eds. A. Sawada and A. Sugamoto (KEK, 1979), p.95; R.N. Mohapatra and G. Senjanović, Phys. Rev. Lett. 44, 912 (1980).

[6] M. Fukugita and T. Yanagida, Phys. Lett. B 174, 45 (1986).

[7] P. Langacker, R. Peccei and T. Yanagida, Mod. Phys. Lett. A 1, 541 (1986); A. Acker, H. Kikuchi, E. Ma and U. Sarkar, Phys. Rev. D 48, 5006 (1993); M. Flanz, E.A. Paschos and U. Sarkar, Phys. Lett. B 345, 248 (1995); L. Covi, E. Roulet and F. Vissani, Phys. Lett. B 384, 169 (1996); M. Flanz, E.A. Paschos, U. Sarkar and J. Weiss, Phys. Lett. B 389, 693 (1996); W. Buchmuller and M. Plümacher, Phys. Lett. B 389, 73 (1996); Phys. Lett. 431 (1998) 354; A. Pilaftsis, Phys. Rev. D 56, 5431 (1997).

[8] E. Ma and U. Sarkar, Phys. Rev. Lett. 80, 5716 (1998). 
[9] C. Wetterich, Nucl. Phys. B 187, 343 (1981); G.Lazarides, Q. Shafi and C. Wetterich, Nucl. Phys. B181, 287 (1981); R.N. Mohapatra and G. Senjanovic, Phys. Rev. D 23, 165 (1981); R. Holman, G. Lazarides and Q. Shafi, Phys. Rev. D27, 995 (1983); G. Lazarides and Q. Shafi, report no hep-ph/9803397.

[10] For instance, R. Foot, H. Lew, X.-G. He, and G.C. Joshi, Z. Phys.C 44, 441 (1989)

[11] A. Zee, Phys. Lett. B 93, 389 (1980).

[12] A. Smirnov,talk given at the 28th International Conference on High Energy Physics (1996),Warsaw,Poland (hep-ph/9611465); N. Gaur, A.Ghosal, E. Ma, P.Roy, Phys. Rev D 58, 7301 (1998).

[13] E. Ma, M. Raidal and U. Sarkar, in preparation.

[14] J.N. Fry, K.A. Olive and M.S. Turner, Phys. Rev. Lett. 45, 2074 (1980); Phys. Rev. D 22, 2953 (1980); Phys. Rev. D 22, 2977 (1980); E.W. Kolb and S. Wolfram, Nucl. Phys. B 172, 224 (1980).

[15] H. Pagels, J.R. Primack, Phys. Rev. Lett. 48, 223 (1982).

[16] M.Yu. Khlopov, A. D. Linde, Phys. Lett. B 138, 265 (1984).

[17] J. Ellis, J. Kim and D.V. Nanopoulos, Phys. Lett. B145, 181 (1984).

[18] J. Ellis, G.B. Gelmini, J.L. Lopez, D.V. Nanopoulos and S. Sarkar, Nucl. Phys. B 373, 399 (1992).

[19] M. Bolz, W. Buchmüller and M. Plumacher, hep-ph/9809381.

[20] M. Kawasaki, T. Moroi, Progr. Theor. Phys. 93 (1995)879; T. Moroi, Ph.D. Thesis (hep-ph/9503210) 
[21] E. Holtmann, M. Kawasaki, K. Kohri and T. Moroi, hep-ph/9805402

[22] D.J.H. Chung, E.W. Kolb and A. Riotto, hep-ph/9809453.

[23] J.C. Pati and A. Salam, Phys. Rev. D 10, 275 (1974); R.N. Mohapatra and J.C. Pati, Phys. Rev. D 11, 566 (1975); R.N. Mohapatra and G. Senjanovic, Phys. Rev. D 12, 1502 (1975); R.E. Marshak and R.N. Mohapatra, Phys. Rev. Lett. 44, 1316 (1980).

[24] M.Plümacher, Nucl. Phys. B 530, 207 (1998). 


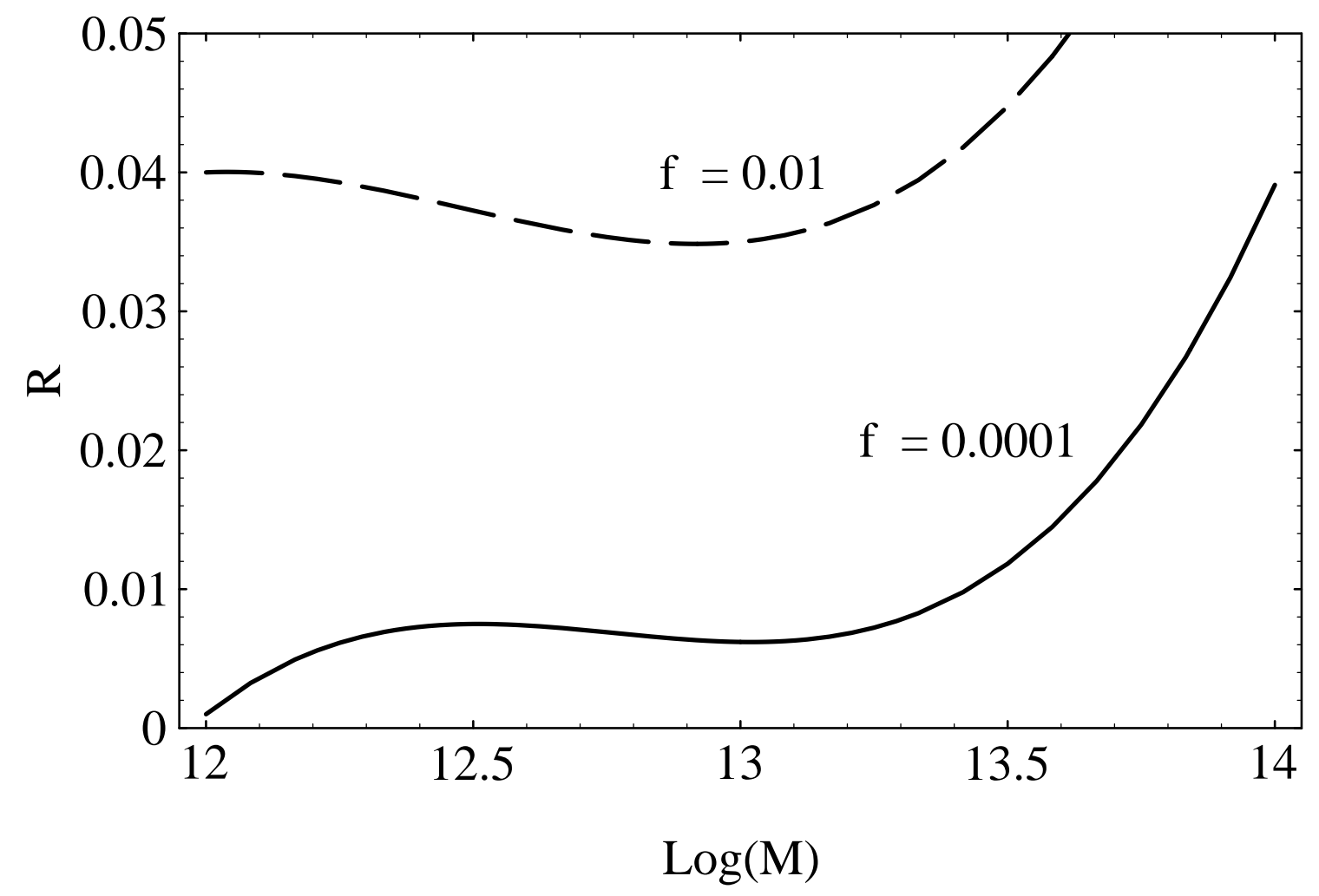

Figure 1: Lepton asymmetry of the universe for different masses of $H_{1}$, when effects of gauge interaction is included. $\mathrm{R}$ is defined as $R=$ $\frac{\left(n_{L} / s\right)_{\text {with }} H_{1}^{\dagger}+H_{1} \rightarrow W_{L}+W_{L}}{\left(n_{L} / s\right)_{\text {without }} H_{1}^{\dagger}+H_{1} \rightarrow W_{L}+W_{L}}$ and $f=f_{i j}^{h}$ 